\title{
Editorial
}

\section{Analysis and interpretation of the electrocardiogram by the computer}

\author{
Velislav Nikolaev Batchvarov
}

St. George's University of London, Cranmer Terrace, London SW17 0RE, United Kingdom

The marriage between computers and electrocardiography (ECG) dates back to the 1960s. Despite its enormous achievements, I am not sure if half a century later it can be considered a very successful marriage from the standpoint of everyday clinical electrocardiography. Numerous deep concepts based on mathematical computerised ECG analysis were developed (e.g. autonomic indices such as heart rate variability/turbulence and deceleration capacity, morphological ECG wave analysis, signal-averaged electrocardiography, beat-to-beat amplitude and morphology variability, etc.) but they all have almost zero impact on everyday ECG-based clinical decision making (the only tentative exclusion is probably signal-averaged electrocardiography). On the other hand, computer readings of important ECG parameters (e.g. heart rate, PR, QRS, QT/QTc interval) today are available on practically every resting 12-lead ECG printout. Clinicians very often take at face value these readings without manual verification. This is clearly a wrong approach that could have dire consequences. Finally, the same clinicians as a rule simply ignore the computer interpretation provided in the same ECG printout (normal ECG vs presence of specific abnormality such as, for example, acute myocardial infarction of certain location). It is beyond the aim of this short text to analyse the root causes of the latter approach; however, every effort to change it and make the automatic ECG interpretation part of the everyday clinical ECG-based decision making should be encouraged.

The study of Fakhri and colleagues [1] in the current issue of this journal seems a right step in this direction. They report an automatic algorithm for assessment of the severity of acute myocardial ischaemia -that is, detection of Grade III ischaemia (ST elevation with terminal QRS distortion) as opposed to Grade I ("hyperacute" Twaveswith no ST elevation) and Grade II ischaemia (ST elevation without terminal QRS distortion). This scoring system for grading of acute ischaemia was introduced by Sclarovsky and Birnbaum more than 20 years ago [2,3] and since then multiple studies (most of them summarised recently in [4]) in have clearly demonstrated that Grade III ischaemia is associated with more rapid progression of necrosis and larger infarct size compared to Grade I or Grade II ischaemia. The authors report a very good agreement between their algorithm and manual expert calculation of the severity grade, and between the automatic scoring and biomarkers of the infarct size, which support the potential clinical application of the algorithm. However, these expectations should be taken with some caution. Firstly, as the authors pointed out, despite all available evidence this grading system is not endorsed by the current guidelines for risk stratification in patients with acute coronary systems and I am not convinced that this is due to "the complex and time consuming nature" of the manual calculation of the score. [1] To my mind a more likely reason is that currently we do not know (due to absence of large prospective trials) how exactly the ischaemia severity grade could/should affect the decision for (or timing of) reperfusion. In addition, the algorithm of Fakhri et al. [1] in its present version may not be universally applicable because it works with representative ("median") ECG complexes calculated from consecutive original ECG complexes in order to reduce the noise level. Median (or average) complexes can be meaningfully constructed only from 
consecutive ECG complexes with identical or very similar morphology. This is often not possible during acute coronary syndromes due to dynamic ECG changes (e.g. frequent ventricular extrasystoles, beat-to-beat QRS-ST changes, etc.). The authors should probably consider possible adaptation of the algorithm to "raw" QRS-ST complexes.

One potential additional benefit of the study of Fakhri et al. [1] is that the concept of their algorithmcould also be useful in other arrythmogenic "J-wave syndromes" such as the Brugada syndrome and malignant early repolarisation. Sclarovsky-Birnbaum Grade 3 ischaemia with the terminal QRS distortion at least visually resembles a severe or malignant "J-wave syndrome" syndrome".

Finally, progress in computerised electrocardiography (including development of automatic algorithms for ECG interpretation) is impossible without the availability of large systematically collected digital ECG databases. Such databases, in my opinion, should be collected in every large hospital. This, however, is not the case for several reasons the most important of them being the fact that clinicians seem perfectly satisfied with visual analysis of "ECG images" (whether paper printouts or digital ECG image files like jpeg, tiff, pdf, etc.). The computer however needs for analysis a "real" digital ECG signal file (that is, a file with the voltage values in each instant of time, i.e. of each sample, in each lead), and not the "image". Enormous treasure of data is lost every day - for example, in the emergency and coronary care units of every large hospital, because the digital signal files are not stored!

As we wrote previously, [5] "image-only" based electrocardiography, without storage of the digital ECG signal, is an obsolete methodology which impedes development of computerised electrocardiography and, ultimately, clinical practice and therefore should be abandoned.

\section{References}

[1] Fakhri Y, Melgaard J, Andersson HB, Schoos MM, Birnbaum Y, Graff C, Sejersten M, Kastrup J, Clemmensen P. Automatic Electrocardiographic Algorithm for Assessing Severity of Ischemia in ST-segment Elevation Myocardial Infarction (in press).

[2] S. Sclarovsky, A.Mager, J. Kusniec, E. Rechavia, A. Sagie, R. Bassevich, et al., Electrocardiographic classification of acute myocardial ischemia, Isr. J. Med. Sci. 26 (1990) 525-531.

[3] Y. Birnbaum, S. Sclarovsky, The grades of ischemia on the presenting electrocardiogram of patients with ST elevation acute myocardial infarction, J. Electrocardiol. 17 (Suppl) (2001) 34.

[4] G.D. Birnbaum, I. Birnbaum, Y. Birnbaum, Twenty years of ECG grading of the severity of ischemia, J. Electrocardiol. 47 (2014) 546-555 (review).

[5] V.N. Batchvarov, E.R. Behr, Clinical utility of computed electrocardiographic leads, J. Electrocardiol. 47 (2014) 281-287. 